We wish to thank the Mond Nickel Company, which lent the pure osmium powder.

E. T. LOUGHER. S. Rowlandos.

George Holt Physics Laboratory, University of Liverpool. Feb. 16.

${ }^{1}$ Bethe, H. A., and Bacher, R. F., Rev. Mod. Phys., 8, 198 (1936). 'Williams, E. J., Proc. Roy. Soc., A, 172, 194 (1939).

"Klemperer, O., "Einführung in die Electronik", p. 272

- Scherrer and Zingg, Helv. Phys. Acta, 12, 283 (1939).

\section{Banded Meson Spectrum and the Rossi Second Maximum}

A vertical counter telescope, designed on the basis of Bhabha's method', was set up as shown in Fig. 1. $5 \cdot 25 \mathrm{~cm}$. of lead used in positions II and III together absorb all but the very high-energy electrons. Such high-energy electrons produce showers in the lead in position II and are cut out from being counted by the shower particles tripping the anti-counters 4 or 5 or both. The effect of side showers on this counter telescope is found experimentally to be negligible ${ }^{2}$. The fact that this arrangement measures the hard component only has also been experimentally verified ${ }^{2}$.

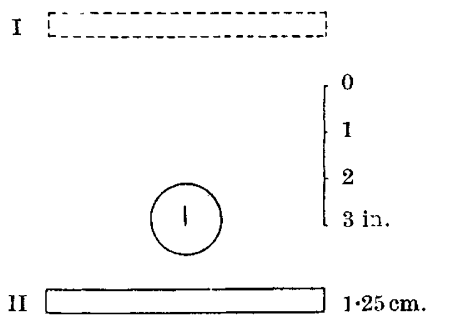

The absorption of mesons was measured by placing lead in position I. This is not objectionable, as it is known that penetrating non-ionizing cosmic rays form a negligible portion of the total intensity ${ }^{3}$. The results obtained after taking every possible care to test the counters, circuits, ets., at the end of each measurement are shown in the ac-
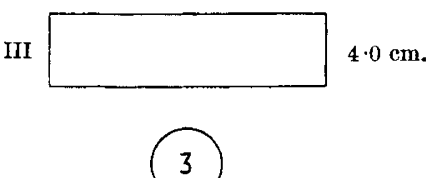

3

Fig. 1. CoUNTERS 1, 2 AND 3 ARE IN COINCIDENCE. COUNTERS 4 AND 5 ARE CONNECTED IN PARALLEL AND IN ANTI-COINCIDENCE WITH COUNTERS 1, 2 AND 3 total amount of lead increases from 21.05 to $23.7 \mathrm{~cm}$. This drop is outside the limits of statistical error of the measurements. The slope of the curve before and after the abrupt drop is the same. The drop in intensity, therefore, appears to be real and

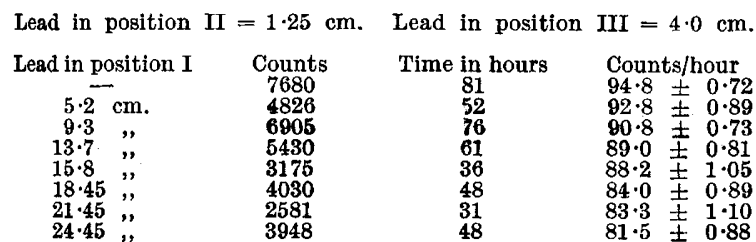

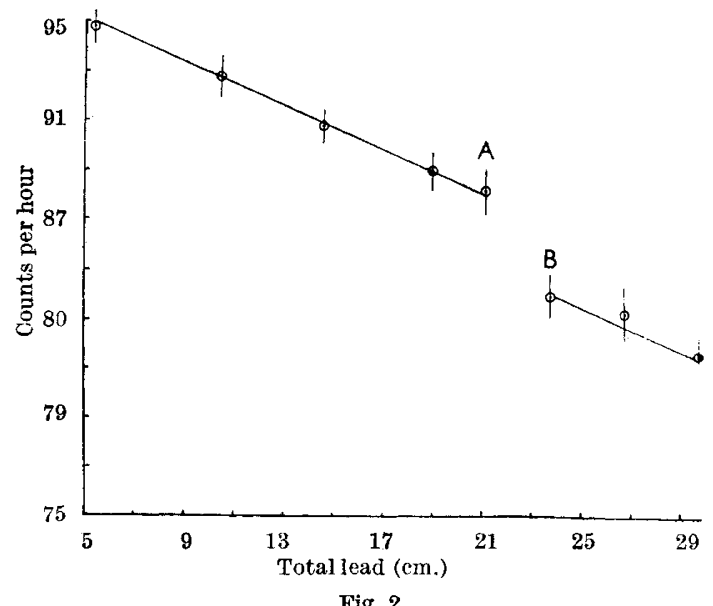

Fig. 2.

not spurious. It occurs when the thickness of lead above the counters 4,2 and 5 lies between $17 \cdot 05 \mathrm{~cm}$. and $19.7 \mathrm{~cm}$. This is the region in which the wellknown Rossi second maximum has been observed by those who get it ${ }^{4}$. Such a drop in the meson absorption curve has not so far been reported by anybody to my knowledge; but its appearance. in this experiment is due to the use of the counter arrangement based on Bhabha's method, which is such as to bring out any existing discontinuities. The interpretation of this experiment, together with the results of further experiments now in progress, will be given in a paper with Prof. Bhabha.

I desire to thank Prof. H. J. Bhabha for his interest and encouragement.

S. V. Chandrashekhar Aiya.

Cosmic Ray Research Unit,

Indian Institute of Science, Bangalore. Jan. 31.

${ }^{1}$ Bhabha, Proc. Inä. Acad. Sci., A, 19, 23 (1944).

In the course of publication.

3 Jánossy and Rochester, Proc. Roy. Soc.. A, 181, 399 (1943). Rossi and Regener, Phys. Rev., 58, 837 (1940).

4 See Proc. Roy. Soc., A, 180, 220 (table I) (1942).

\section{Base Electrolytes for Use in Polarographic Determinations}

IN the course of recent research on the application of the polarographic method to the routine analysis of magnetic materials of the 'Permalloy' type, we have found several new base electrolytes which offer considerable advantages for quantitative polarography. They are characterized by the very satisfactory shape of the 'waves', in general free from disturbing maxima, which they yield with a number of metal ions.

Our main problem at the commencement of this work was the determination of iron (12-18 per cent) and molybdenum (2-5 per cent) in the presence of more than 70 per cent nickel and some copper, manganese, etc. We have succeeded in devising and applying our methods to give extremely rapid and accurate routine determinations of these elements in particular, so that to date some six thousand individual determinations have already been made, with a considerable saving of time.

The sample is dissolved in a sulphuric acid-nitric 